artigstem Schaffen und Gestalten. Und als Unterton - ohne daß davon die Rede war - nur schon durch die Hingabe der Institutsvorsteher und ihrer Helfer, das Vertrauen in uns Mittelschullehrer, daß auch wir unsern Mann stellen. Seien wir dessen würdig. Dank den Herren Dozenten für ihre Bemühungen und Dank dem Vorstand für die Organisation der vielseitig anregenden Tagung.

\title{
DIE SCHWEIZ I955 - EIN LANDESKUNDLICHER RÜCKBLICK
}

\author{
P. KöCHLI
}

Witterungsverlauf. Die mittleren Temperaturverhältnisse für 1955 weichen wenig vom Durchschnitt ab. Das Jahr war im gesamten um einige Zehntelsgrade zu warm. Abgesehen vom Dezember 1955 (in den Niederungen $4-6^{\circ} \mathrm{C}$, in der Höhe ungefähr $2^{\circ} \mathrm{C}$ zu warm) wiesen auch die einzelnen Monate keine größeren Temperaturabweichungen auf. Der Witterungsverlauf wird besser durch die Niederschlagsverhältnisse charakterisiert. Es war ein ausgesprochener Wechsel von trockenen zu nassen Perioden zu verzeichnen. Auf einen niederschlagsreichen Winter folgte eine mehr als zweimonatige Trockenzeit im Frühling. Von Mai bis anfangs August herrschte eine ausgesprochene Regenzeit, die ihren Höhepunkt im gewitterreichen Juli fand. Hierauf setzte eine Trockenperiode ein, die bis zu einer erneuten Umstellung der Großwetterlage auf den zonalen Typus im Dezember andauerte und den unerwarteten und gefährlichen Tiefstand unserer Flüsse zu Winterbeginn bewirkte. An klimatischen Besonderheiten seien erwähnt:

Der Winter 1954/55 verzeichnete die merkwürdige Tatsache von vier scharfen Kaltlufteinbrüchen in fast gleichen Abständen von anderthalb Monaten. Der erste erfolgte Mitte November, der zweite auf Jahreswechsel, der dritte Mitte Februar (kälteste Winterperiode vom 12.2.-13.3.55), der letzte Ende März. Da die Schweiz im Januar und Februar im Grenzbereich der Warmluft zur Kaltluft lag, fielen sehr große Niederschläge, die oberhalb $1500 \mathrm{~m}$ zu einer hohen Schneedecke führten. In den Voralpen und im Mittelland verhinderte dagegen das häufige Tauwetter ein starkes Anwachsen der Schneedecke, die im Frühjahr auch entsprechend rasch verschwand. Die Wetterlage des Sommers bildete sich Ende April heraus. Ein stabiler, warmer Hochdruckkeil schob sich von den Azoren über Großbritannien nach Südskandinavien vor. Mitteleuropa war in den Monaten Juni bis August praktisch von den Einbrüchen maritimer Luft abgeschnitten. Dagegen drangen immer wieder mit nordöstlichen Strömungen feuchte Kaltlufttropfen nach Zentraleuropa, die bei der hier herrschenden flachen Druckverteilung sich nur langsam verschoben und mit ihrer instabilen Schichtung starke Bewölkung und häufige, wiederkehrende, zum Teil heftige Gewitter veranlaßten. Die Zahl der Gewittertage 1954 zu 1955 war 1:6 (13732 Hagelschadenmeldungen, 4,65 Millionen Fr. Entschädigungen).

Wirtschaftliche Verhältnisse. Die Schweiz verzeichnete wiederum ein Jahr guter Beschäftigung, die hauptsächlich durch die hohe Investitionstätigkeit, die Bauindustrie und die lebhafte Ausfuhrtätigkeit getragen wurden.

a) Landwirtschaft und Forstwirtschaft. Ertragsmäßig war 1955 ein mittleres Jahr. Die Landwirtschaft nimmt an der allgemeinen Konjunktur wenig Anteil. Der Preisindex der landwirtschaftlichen Erzeugnisse stieg innert Jahresfrist um einen Punkt auf 1oo,2 Punkte im Oktober $1955(1948=100)$, der Index der landwirtschaftlichen Produktionsmittel um 2,6 Punkte auf 107,2 Punkte. Es ist nicht anzunehmen, daß der Kaufkraftverlust vollständig durch eine Produktivitätssteigerung ausgeglichen werden konnte.

Die Fläche des offenen Ackerlandes hat um weitere $2 \%$ zugenommen und beträgt nun annähernd 278000 ha. Damit ist das vorläufige Ziel von 280000 ha beinahe erreicht. Die Erweiterung auf 300000 ha soll erst nach dem Bau der zweiten Zuckerfabrik vorgenommen werden. Vom offenen Ackerland entfallen $65 \%$ auf Getreide, 21,1\% auf Kartoffeln, 4,3\% auf Gemüse, 7,3\% auf Wurzelgewächse und $2 \%$ auf andere Ackerfrüchte Die Kartoffelernte betrug bloß $1100000 \mathrm{t}$ (1954: $1395200 \mathrm{t})$; die Zuckerrübenernte war rund 10\% kleiner (1955: $200000 \mathrm{t}$ ), der Zuckergehalt dagegen hoch (15,9-16\%). Der Rebbau beanspruchte 13139 ha oder 134 ha weniger als im Vorjahr. Die Anbaufäche beträgt in der deutschen Schweiz noch 1914 ha, in der welschen Schweiz 9420 ha und im Tessin und Misox 1802 ha. 11818 ha waren mit der europäischen Rebe, 1320 ha mit Direktträgerkreuzungen bepflanzt. $66,4 \%$ war mit weißem, 33,6\% mit rotem Gewächs bestanden. Die Weinernte betrug $800966 \mathrm{hl}$, davon waren 607 o74 hl Weißwein und 193892 hl Rotwein. Die Hektarerträge erreichten im Mittel $63,8 \mathrm{hl}$ gegenüber $54,8 \mathrm{hl}$ im Vorjahre. Die Hektarerträge sind durchschnittlich in der Westschweiz doppelt so hoch als in der Ostschweiz, was auf Klima und andere Rebschnitte zurückgeführt wird. 
b) Der Außcnhandel zeigt folgende Entwicklung:

\begin{tabular}{lcccc} 
& \multicolumn{2}{c}{ Einfuhr } & \multicolumn{2}{c}{ Ausfuhr } \\
& 1954 & 1955 & 1954 & 1955 \\
\hline Wagen zu 10 t & 1016934 & 1119061 & 80833 & 85064 \\
Wert in Mio Fr. & 5591,6 & 6401,2 & 5271,5 & 5622,2
\end{tabular}

Defizit des Warenverkehrs: 779,o Mio Franken.

Von dieser Gütermenge wurden über die Rheinhäfen Basel 4,1 Mio t eingeführt (36,6\%), 456,446 t $(53,6 \%)$ ausgeführt.

Der Außenhandel weist folgende räumliche Verteilung und Verschiebungen auf:

1954 in $\%$ Einfuhr 1955 in $\%$ Verän- $\quad 1954$ in $\% \quad \begin{gathered}\text { Ausfuhr } \\ 1955 \text { in } \% \text { Verän- }\end{gathered}$ in Mio Fr. $\quad$ in Mio Fr. derung in $\%$ in Mio Fr. in Mio Fr. derung in $\%$

\begin{tabular}{lrrrrr|rrrrr}
\hline Europa & 3846,6 & 68,82 & 4547,3 & 71,04 & $+2,22$ & 3109,4 & 58,99 & 3326,7 & 59,18 & $+0,19$ \\
Nordamerika & 860,1 & 15,38 & 983,3 & 15,37 & $-0,01$ & 731,3 & 13,87 & 753,1 & 13,39 & $-0,48$ \\
Zentralamerika & 123,8 & 2,21 & 117,8 & 1,84 & $-0,37$ & 110,0 & 2,09 & 112,1 & 1,99 & $-0,10$ \\
Südamerika & 297,4 & 5,31 & 234,8 & 3,66 & $-1,65$ & 397,3 & 7,54 & 403,6 & 7,18 & $-0,36$ \\
Afrika & 192,3 & 3,44 & 224,1 & 3,50 & $+0,06$ & 280,4 & 5,32 & 326,5 & 5,81 & $+0,47$ \\
Asien & 235,8 & 4,21 & 261,7 & 4,09 & $-0,12$ & 522,3 & 9,90 & 577,4 & 10,27 & $+0,37$ \\
Australien, & 35,2 & 0,63 & 32,2 & 0,50 & $-0,13$ & 120,8 & 2,29 & 122,8 & 2,18 & $-0,11$ \\
Ozeanien & & & & & & & & & &
\end{tabular}

Europa ist für die Einfuhr noch wichtiger geworden als 1954; die Ausfuhr dagegen ist weniger gestiegen, so da $\beta$ aus dem europäischen Handel ein vergrößertes Defizit entstanden ist. Der Güteraustausch mit den einzelnen überseeischen Staaten zeigt eine unterschiedliche Entwicklung. Die bedeutendsten Zunahmen im Export entfallen auf Aegypten, Argentinien, Japan und Kanada. Mehr eingeführt haben wir aus den Vereinigten Staaten, China und Japan, wesentlich weniger aus Argentinien, Brasilien und Aegypten.

c) Elektrizitätswirtschaft (Hydrographisches Jahr: 1. Oktober 1954-30. September 1955). Dieses Jahr sind in zahlreichen Zeitschriften und Zeitungen umfangreiche Abhandlungen über die Elektrizitätswirtschaft erschienen (Neue Zürcher Zeitung: 3. Juni 1955, Bund: 1o. September 1955). Erzeugung und Verbrauch zeigen folgende Entwicklung:

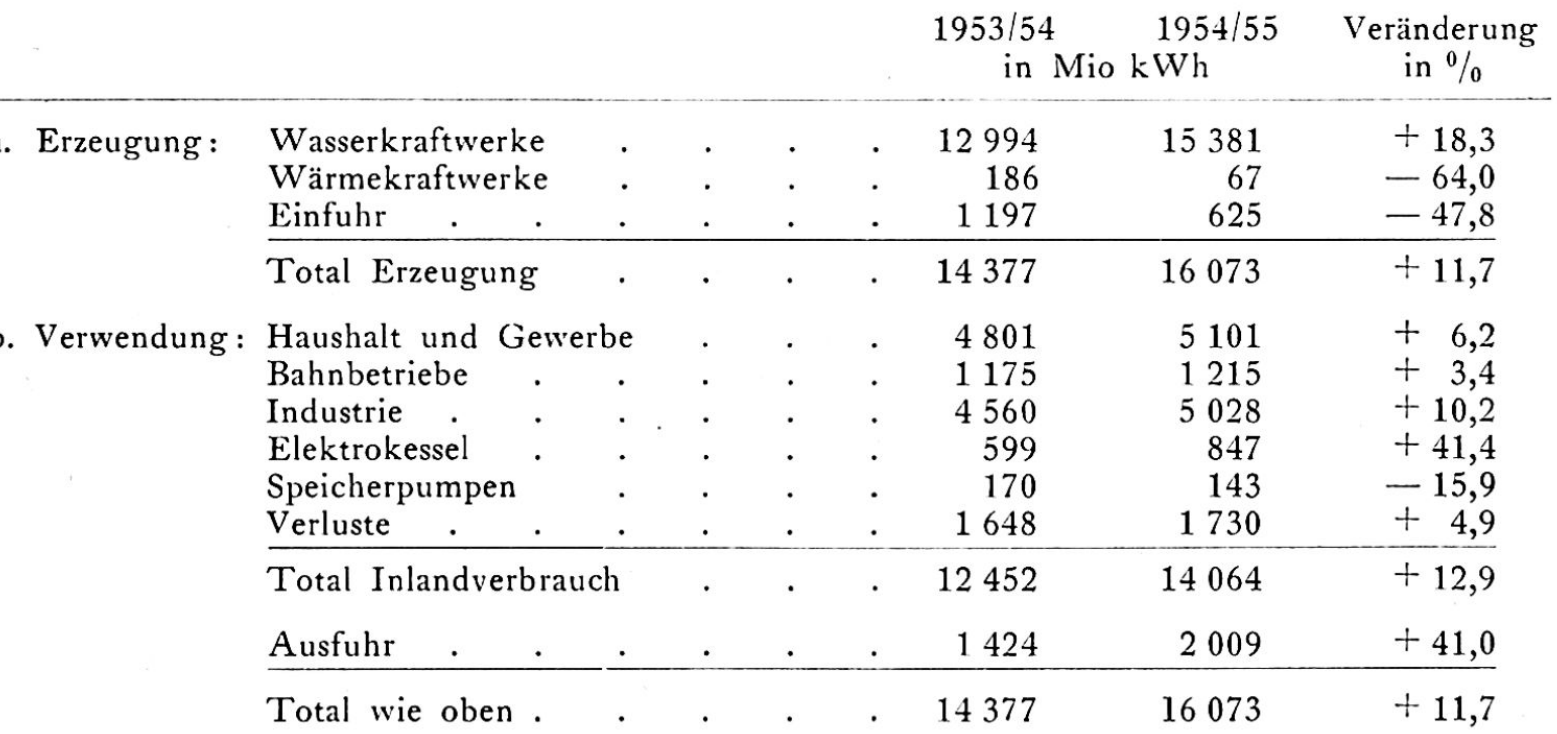

Infolge der technischen Entwicklung schätzt man die mögliche Energieproduktion der Schweiz jährlich auf 30 Milliarden $\mathrm{kWh}$. Darüber gibt eine Übersicht der eidg. Behörden Auskunft: 


\begin{tabular}{|c|c|c|c|c|c|c|c|}
\hline \multirow{3}{*}{ Werke } & \multirow{3}{*}{$\begin{array}{c}\text { Höchstleistung } \\
\text { (Generator) } \\
\text { Mio kW }\end{array}$} & \multicolumn{2}{|c|}{ Speicher } & \multirow{2}{*}{\multicolumn{3}{|c|}{$\begin{array}{l}\text { Mögliche mittlere } \\
\text { Energieproduktion } \\
\text { Mio } \mathrm{kWh}\end{array}$}} & \multirow{3}{*}{$\begin{array}{l}\text { Ungefähre } \\
\text { Anlagekosten } \\
\text { ohne Verteil- } \\
\text { anlagen } \\
\text { Mio Fr. }\end{array}$} \\
\hline & & \multirow{2}{*}{$\begin{array}{l}\text { Nutz- } \\
\text { inhalt } \\
\text { Mio } \mathrm{m}^{3}\end{array}$} & \multirow{2}{*}{$\begin{array}{c}\text { Energie } \\
\text { inhalt } \\
\text { Mio kWh }\end{array}$} & & & & \\
\hline & & & & Winter & Sommer & $\mathrm{Jahr}$ & \\
\hline $\begin{array}{l}\text { im Betrieb } \\
\text { im Bau }\end{array}$ & $\begin{array}{l}3,506 \\
0,919\end{array}$ & $\begin{array}{r}1136 \\
522\end{array}$ & $\begin{array}{l}1862 \\
1540\end{array}$ & & $\begin{array}{l}8442 \\
1006\end{array}$ & $\begin{array}{r}15298 \\
2842 \\
\end{array}$ & $\begin{array}{l}2834 \\
1498\end{array}$ \\
\hline Total & 4,425 & 1658 & 3402 & 8692 & 9448 & 18140 & 4332 \\
\hline $\begin{array}{l}\text { Projekte } \\
\text { kleine Anlagen }\end{array}$ & $\begin{array}{l}3,293 \\
0,067\end{array}$ & 1604 & 4086 & $\begin{array}{r}6566 \\
142\end{array}$ & $\begin{array}{r}5022 \\
130\end{array}$ & $\begin{array}{r}11588 \\
272 \\
\end{array}$ & $\begin{array}{r}4875 \\
33\end{array}$ \\
\hline & 7,785 & 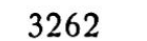 & 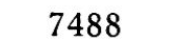 & 5400 & 4600 & 30000 & 924 \\
\hline
\end{tabular}

In diesem Zusammenhang ist es aufschlußreich, den mengenmäßigen Anteil der einzelnen Energieträger an der Deckung des Nutzenergiebedarfes (Jahr 1951) festzuhalten (Bericht des Komitees für Energiefragen im Schweizerischen Nationalkomitee der Weltkraftkonferenz):

\begin{tabular}{lc} 
Einheimisch: & Anteil in \% des Gesamtverbrauchs \\
\hline Holz & 10,5 \\
Hydroelektrizität & 28,5 \\
Import : & \\
Kohle und Gas & 41,3 \\
Heizöl & 16,5 \\
Benzin und Dieselöl & 3,2 \\
\hline Insgesamt & 100,0
\end{tabular}

Infolge der Hochkonjunktur nimmt der Verbrauch von Hydroelektrizität, Heizöl und Benzin stark zu, was einen raschen Ausbau der Kraftwerke veranlaßt. 1955 sind folgende Werke in Betrieb gekommen: Marmorera, Ernen, Les Clées II und Birsfelden; dazu kamen bedeutende Um- und Neubauten bei den Kraftwerken Bellefontaine, Oberhasli, Ritom, Barberine, Bisistal und Isental. Die mittlere mögliche Energieproduktion stieg dadurch um 658,+ Mio $\mathrm{kWh}$ an. Gegenwärtig sind folgende 17 Kraftwerke im Bau: Rheinau (Fertigstellung 1956), Grande Dixence, 1. Phase (1956), Alpnach (1957), Simplonwerke (1957), Arnensee (1957), Lienne (1958), Maggia, 1. Etappe (1958), Melchsee-Frutt (1958), Pallazuit (1958), Ritom, Zuleitung der Unteralpreuß (1958), Zervreila-Rabiusa (1958), Gougra-Navizance (1959), Mauvoisin (1959), Göscheneralp (1961) und Bergeller-Kraftwerke (1961/62).

Über den Zusammenhang zwischen Wasserkraftnutzung und Industrieentwicklung orientiert das Zentralbüro der Schweizerischen Vereinigung für Landesplanung wie folgt: «Die erste Industrialisierungsphase baute auf einer ausgedehnten Heimindustrie auf. An Stelle der Heimindustrie trat dann weitgehend die Fabrik. Sie war zuerst an den Ort der Wasserkraft gebunden; denn eine Umwandlung in elektrische Energie und deren Fortleitung kannte man noch nicht.

Mit der Umformung der mechanischen Energie in elektrische Energie und der Übertragung auf weite Entfernungen beginnt das Zeitalter der Mittel- und Großkraftwerke. Ein neuer, entscheidender Standortsfaktor trat auf, die günstige Transportlage. Die Industrie setzte sich nun längs der großen Eisenbahnlinien fest... So entwickelte sich dort, wo eine bedeutende Wasserlaufindustriezone eine bedeutende Eisenbahnlinie schneidet, ein Industrieort. Es ist dies die dritte Industrialisierungsphase.

Mit der Konzentrierung der Industrie ergaben sich aber verschiedene, wohlbekannte Nachteile. So hat denn etwa seit zehn bis fünfzehn Jahren eine neue, vierte Industrialisierungsphase eingesetzt, die Rückkehr der Industrie aus den Zentren in ländliche Industrieregionen. Welche Rolle die über weite Flächen verteilbare elektrische Energie bei diesem Vorgange spielt, kann man sich leicht vorstellen. Auch daraus ersieht man, welche große Bedeutung unsere Wasserkräfte in staatspolitischer Hinsicht haben, nämlich für eine Dezentralisierung und möglichste Ausgleichung des Wirtschaftspotentials, wie sie der demokratischen und föderativen Organisation unseres Landes angemessen ist. (Vergleiche dazu die Industriekarten der Schweiz des Verlages Kümmerly \& Frey).

d) Verkchr: Im Netz aller Bahnen ergaben sich nur unwesentliche Veränderungen. Die Zahl der Reisenden stieg bei den Schweiz. Bundesbahnen auf

ihr Güterverkehr auf

Der Ausbau der eidgenössisch konzessionierten Pendel-, Gondel- und Sesselbahnen geht rasch vorwärts und erfaßt immer neue Landesgegenden. 
Der Straßenverkehr stieg weiterhin sprunghaft an. Der Motorfahrzeugbestand am 3o. September 1955 war:

\begin{tabular}{lrrr} 
Fahrzeugart & 1954 & 1955 & Veränderung in $\%$ \\
Autobusse, Autocars & 2432 & 2502 & $+2,8$ \\
Personenwagen & 237510 & 270821 & $+14,0$ \\
Personenwagen für Warentransport & 6903 & 8696 & $+25,9$ \\
Lieferwagen & 14949 & 15883 & $+6,2$ \\
Lastwagen & 24641 & 26038 & + \\
Spezialwagen & 2637 & 2801 & $+6,2$ \\
Gewerbliche Traktoren & 1106 & 1149 & $+3,8$ \\
Motorfahrräder & 45776 & 59201 & $+29,3$ \\
Roller & 67937 & 76654 & $+12,8$ \\
Motorräder & 80092 & 80586 & $+0,6$ \\
\hline Total & 483983 & 544331 & $+12,4$
\end{tabular}

Die Zahl der Fahrräder ist leicht zurückgegangen. Der wachsende Straßenverkehr zwingt zu einem raschen Ausbau des Straßennetzes. Die Pläne für die Autobahnen und ihre Zubringerstraßen sollen 1956 veröffentlicht werden. Daneben sind eifrige Aussprachen für Straßenverkehrstunnels zur Durchstoßung der Alpen im Gange, damit die Schweiz ihre Stellung als Transitland behalten kann.

c) Verschicdenes: Um einige Fragen, die letztes Jahr stark im Vordergrund gestanden sind, ist es etwas stiller geworden, so um die zweite Juragewässerkorrektion, den transhelvetischen Kanal und die Erdölbohrung in Altishofen, die nur ein unsicheres Ergebnis gezeitigt hat. Dagegen war die Bohrung nach der Thermalquelle Zurzach äußerst erfolgreich.

f) Ausblick für 1956. Soweit sich bis jetzt beurteilen läßt, wird eine weitere langsame Zunahme in der Industrialisierung zu erwarten sein. Dabei bestrebt man sich, die Auflockerung zu fördern und vor allem auch Berggebiete zu berücksichtigen, um den Bergbauern ihr Los zu erleichtern, und die Abwanderung hintanzuhalten. Landschaftliche Veränderungen werden sich vor allem durch den Kraftwerkbau einstellen.

Qucllen: Die Volkswirtschaft 1955/56, Bern, Berichte der Schweiz. Bankgesellschaft 1955, Bulletin des SEV 1955, führende Tageszeitungen u. a. m. Ferner sei zahlreichen eidgenössischen und kantonalen Ämtern für bereitwillige Auskunft bestens gedankt.

\section{DIE NEUE KULTURLANDKARTE, DER SCHWEIZ ARTHUR DÜRST}

Die Reihe der thematischen Karten der Schweiz hat durch die von Prof. Dr. F. T. WAHLEN angeregte und von den Ingenieuren E. Neuenschwander und M. Hauswirth vom Produktionskataster bearbeiteten Kulturlandkarte 1 erneut eine erfreuliche Bereicherung erfahren.

Das für die Gestaltung der Karte notwendige Material lieferte der 1938 vom Bundesrat als diagnostische Maßnahme angeordnete landwirtschaftliche Produktionskataster, der die ackerbaulichen Möglichkeiten aufzeigen und die Bestimmung der optimalen Anbaufläche erlauben sollte. Die unter der Leitung von Prof. Dr. F. T. WAHLEN durch Experten des Bundes gemeindeweise durchgeführten Aufnahmen, die durch den Ausbruch des Weltkrieges zu einem dringenden Anliegen des Eidg. Kriegsernährungsamtes wurden, lagen ungefähr im Jahre 1942 für den Jura, das Mittelland und die Voralpen vollständig vor und erfuhren in der Folge durch die Erfassung der alpinen Gebiete ihre Ergänzung. Gründliche Revisionen nach Beendigung des Krieges trugen den inzwischen eingetretenen Veränderungen Rechnung, sodaß der Inhalt der Karte ungefähr dem Stand des Jahres 1948 entsprechen dürfte. Der auf den Blättern anzutreffende Vermerk «Ausgabe 1951》 steht in keiner Beziehung zum Dargestellten, sondern gehört zur Gemeindekarte der Schweiz, die, da die Erhebungen des Produktionskatasters gemeindeweise vorlagen, mit Recht als Kartengrundlage gewählt wurde.

Die Begriffe Kulturland im engeren Sinne, Weideland, Streueland, Wald und Unproduktiv, wie sie bei der in der Kulturlandkarte erfolgten Fünfteilung Anwendung finden, werden

1 Kulturlandkarte der Schweiz $1: 200000$ in vier Blättern. 1952/54. Herausgegeben von der Abteilung für Landwirtschaft im Eidg. Volkswirtschaftsdepartement. Aufnahmen und Bearbeitung durch den Produktionskataster. Druck durch die Eidg. Landestopographie. Die Karten können bezogen werden bei der Abteilung für Landwirtschaft, Laupenstraße 25, Bern. Preise : Einzelblatt Fr. 10.- ; Blätter 1-4 zusammen Fr. 36.-. Ganze Karte auf Leinwand aufgezogen und mit Stäben versehen Fr. 56.- Spezialpreis für Schulen Fr. 48.- 